\title{
Empirical Analysis of the Spread of University Students' Amative Behavior
}

\author{
Xiaoling $\mathbf{Y u}^{1}$, Zhen Jia ${ }^{1,2}$, Cuiping Zhang ${ }^{1}$ \\ ${ }^{1}$ College of Science, Guilin University of Technology, Guilin, China \\ ${ }^{2}$ Guangxi Key Laboratory of Spatial Information and Geomatics, Guilin, China \\ Email: 1243482738@qq.com
}

Received May 16, 2013; revised June 16, 2013; accepted June 25, 2013

Copyright (C) 2013 Xiaoling Yu et al. This is an open access article distributed under the Creative Commons Attribution License, which permits unrestricted use, distribution, and reproduction in any medium, provided the original work is properly cited.

\begin{abstract}
We used a questionnaire to obtain data about 664 university students' amative behavior in a campus. Then we studied dissemination rules of university students' amative behavior on campus social network. We found the amative behavior changes over time by focusing on the analysis of love group's influence to single people and single group's affected degree. Meanwhile, we compared the influence of single and multiple social relationships to the spread of amative behavior, and the result shows diversity of social relationships is a significant effect factor in spreading process.
\end{abstract}

Keywords: Social Network; Amative Behavior; Propagation Behavior

\section{Introduction}

Complex network is a new issue in recent years. From regular network at first, random network which is built by mathematicians in 1950s [1], to two pioneering work in 1990s-WS network built by Watts and Strogatz in 1998 [2] and NW network built by Newman and Watts [3], complex network has gotten people's attention. Many structures in real world can be intuitively represented by complex networks. A typical network consists of lots of vertexes and edges which contact two vertexes. Vertexes in a network represent different individuals in the real system, and every edge indicates relation between two vertexes. Usually, two vertexes are connected by an edge when some certain relationship between them; unconnected when there is no relation. We consider two vertexes connected by an edge are next in a network. For example, nervous system is regarded as a network, in which lots of nervous cells are connected by nerve fiber [4]. There are many networks around us, like electricity network [3], social network [3,5], traffic network [6]. Complex network attracts the interest of scholars owing to its unique charms and shows its exuberant vitality.

In real world, people and relationship between them constitute networks, which are called social network. Common examples are interpersonal communication network, e-mail network, online social networks and so on. Social networks have attracted great interest in recent years, largely because of their likely relevance to various social processes [6], such as information processing [7], distributed search [8], and diffusion of social influence [9].

The process of social contagion is different from the process of disease transmission. In the spread of disease, when a healthy node contacts an infected node, the probability of infection has something to do with the contagiousness of the disease, the healthy node's resistance to disease and so on, which are objective. However, in the process of social contagion, when inactive nodes contact active nodes, they decide to participate or not. It means, the process of social contagion may be more to do with subjective factor. The uncertainty of individuals' decisions determines the complexity of social contagion. Although decisions of individuals possess uncertainty, is there statistical law when behavior spreads in social network constituted by many individuals?

\section{Data and Campus Social Network}

We investigated some senior students' amative behavior in a university, and tried to find dissemination rules of amative behavior on social network. We used systematic sampling to obtain a sample which has 664 senior students. Next, we focused on four kinds of social relationships (classmates, countrymen, roommates and members of the same association) between them and built campus social network model.

With the help of UCINET, we got statistical charac- 
teristics of four sub-networks and the whole network, and we compared them with statistical characteristics of fullscale random network:

According to Table 1, we held the opinion that four sub-networks and the whole network have small world characteristic.

From the first semester, 664 nodes began a love relationship one by one. Until the senior last semester, all nodes have experience in love. Once a node started a love relationship, it became an infected node whether it broke up or not during this period, and we call it love node. Every semester is a stage, so there are seven stages from the freshman last semester to the senior last semester, and in first stage there were 126 infected nodes. After seven stages, all nodes became infected nodes.

\section{Statistical Analysis of the Spread of Amative Behavior in Each Subnet and the Whole Network}

In order to compare the different impact of different relations on the spread of love behavior, we extracted four kinds of relationships between students in the campus social network. They were classmates, countrymen, roommates and members of the same association, constituting the subnet of the overall campus social network. We study the four subnets from two aspects, the influence strength of love node's affecting on its neighbor nodes, and the influence strength of the non-love node's being affected by its neighbor nodes. But when it comes to the overall network, in addition to the above two aspects, the density of love nodes out of one's total neighbor nodes is also taken into account.

\subsection{Influence Strength of Love Node's Affecting on Its Neighbor Nodes}

In order to analyze and compare the different strengths of impacts, caused by different neighbor nodes, on the spread of love, we gave the formula to calculate the influence strength caused by each single relationship between neighbor nodes.

In the $i$ th stage, within the scope of a single relationship (namely in a certain relationship subnet), if node A is a love node, then its influence strength to its neighbor node (in the corresponding subnet) is given by (1). Here

Table 1. Comparison of statistical characteristics.

\begin{tabular}{ccc}
\hline Web & Average shortest path length & clustering coefficient \\
\hline Random & 1.816 & 0.184 \\
Classmate & 0.935 & 0.5 \\
Countrymen & 0.967 & 0.5 \\
Roommate & 0.999 & 0.5 \\
Association & 0.998 & 0.5 \\
Whole & 0.928 & 0.365 \\
\hline
\end{tabular}

$$
x_{A i}=N_{A, i+1} / N_{A i^{\prime}} .
$$

in which $x_{A i}$ means node $A$ 's influence strength on its neighbors in the ith stage, $N_{A, i+1}$ means the number of newly added love nodes among the neighbor nodes of node $\mathrm{A}$ in the $i+1$ th stage, and $N_{A i^{\prime}}$ represents the total number of non-love nodes among the neighbors of node $\mathrm{A}$ in the ith stage.

In the ith stage, within the scope of a single relationship (namely in a certain relationship subnet), the average influence strength of love nodes to other nodes (in the corresponding subnet) is given by (2). Here

$$
\bar{x}_{i}=x_{i} / N_{l i} .
$$

in which $\bar{x}_{i}$ means the average influence strength of love nodes in the corresponding subnet in the ith stage, $x_{i}$ means the summation of the influence strength of all the love nodes in the certain subnet in the ith stage, and $N_{l i}$ represents the total number of all the love nodes in the ith stage.

In the ith stage, when it comes to the overall network, the average influence strength of all the love nodes (in the whole network) to other nodes is given by (3). Here

$$
\bar{X}_{i}=X_{i} / N_{l i} .
$$

in which $\bar{X}_{i}$ means the average influence strength of all the love nodes in the whole network in the ith stage, $X_{i}$ means the summation of the influence strength of all the love nodes in the whole network in the ith stage, and $N_{l i}$ represents the total number of all the love nodes in the ith stage.

Formula (1) and Formula (2) are used to calculate according to the four subnets respectively, while Formula (3) is the comprehensive calculation of the whole campus social network.

We got the data about the influence strength of love nodes to other nodes by the Formula (1). After further analysis, we got the influence strength in every stage and every subnet. The results are shown in Figure 1.

Figure $\mathbf{1}$ is divided with dash line into four parts: Classmates subnet (D), Countrymen subnet (N), Roommates subnet $(\mathrm{R})$ and members of the same association subnet (C).The abscissa, Di means the ith stage of the classmates subnet, $\mathrm{Ni}$ means the $i$ th stage of the countrymen subnet, $\mathrm{Ri}$ means the ith stage of the roommates subnet and $\mathrm{Ci}$ mans the ith stage of the association subnet $(i=1,2,3,4,5,6)$. From Figure 1, we can see that the influence strength of love nodes shows a rising trend as time goes. It indicates that love nodes gradually increasing influence on not love nodes, and this conclusion can be deduced from Figure 1 also. The denominator $N_{A i^{\prime}}$ represents the total number of not love nodes among the neighbors of node $A$, and it reduces over time. The denominator is getting smaller and smaller, so the influence 


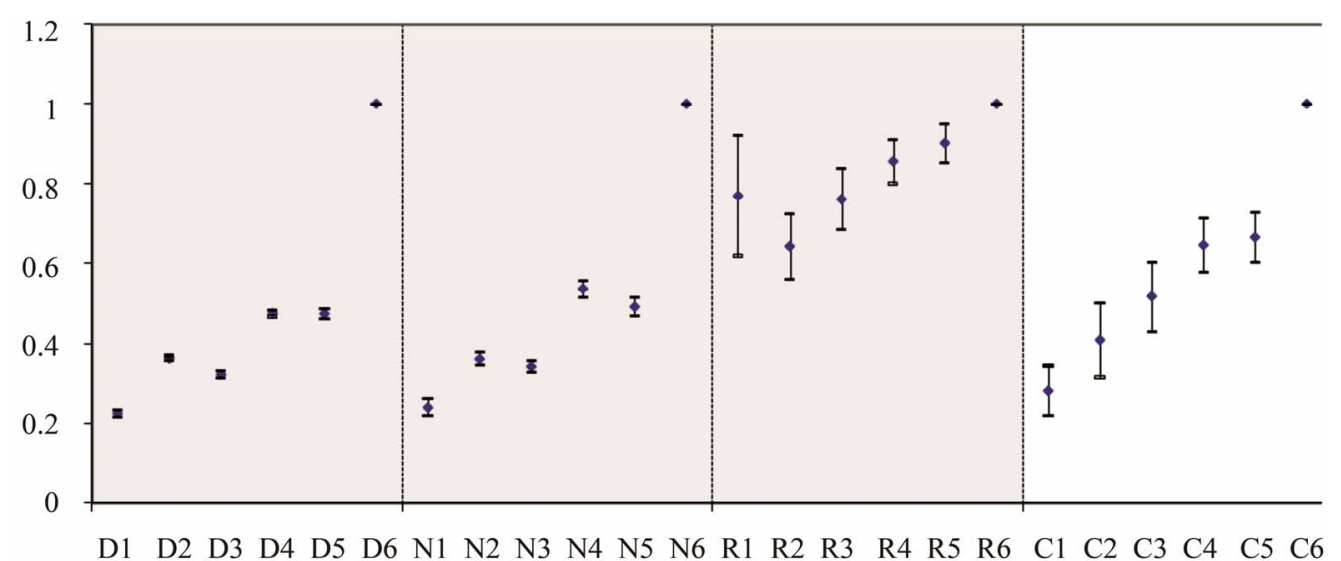

Figure 1. Influence strength of love nodes. Error bars represent 95\% confidence intervals.

strength becomes larger and larger. Judging from the confidence interval span, the confidence interval length of classmate subnet is very short, and it is the same with the countrymen subnet. This indicates that the value of any node's influence strength is more concentrated in every stage, while the value of roommate network and association network is more dispersed. We consider that this is related to the density of the network, as is shown in Table 2, with greater density, the value is more concentrated, on the contrary, the value is more disperse with smaller density.

According to Formula (2) and Formula (3), we got Figure 2.

As is shown in the figure above, both in each subnet and the whole network, the tendency of the average influence strength is basically consistent, and substantially shows a rising trend as time goes. It has an important guiding significance for our students work: during the university, we should pay close attention to the behavior of love students, understand their view of love and mental state. If someone feels confused or has improper view of love, we should give him a depth, honest communication, helping him out of emotional difficulties or misunderstanding. We should also prevent improper views and behaviors of love affect other students, who don't fall in love. Students who have fallen in love, with a positive attitude, can bring good effects to others.

\subsection{Influence Strength of Non-Love Node's Being Affected by Its Neighbor Nodes}

In order to analyze and compare the different strengths of impacts on non-love nodes, caused by different love neighbors, on the spread of love, we gave the formula to calculate the influence strength of non-love nodes, caused by each single relationship between neighbor nodes.

Within the scope of a single relationship (namely in a certain subnet), if node $B$ is a non-love node in the ith stage, and it becomes a love node in the $i+1$ th stage,
Table 2. Network density.

\begin{tabular}{ccccc}
\hline Subnet & Classmate & Countrymen & Roommate & Association \\
\hline Density & 0.065 & 0.033 & 0.0011 & 0.0022 \\
\hline
\end{tabular}

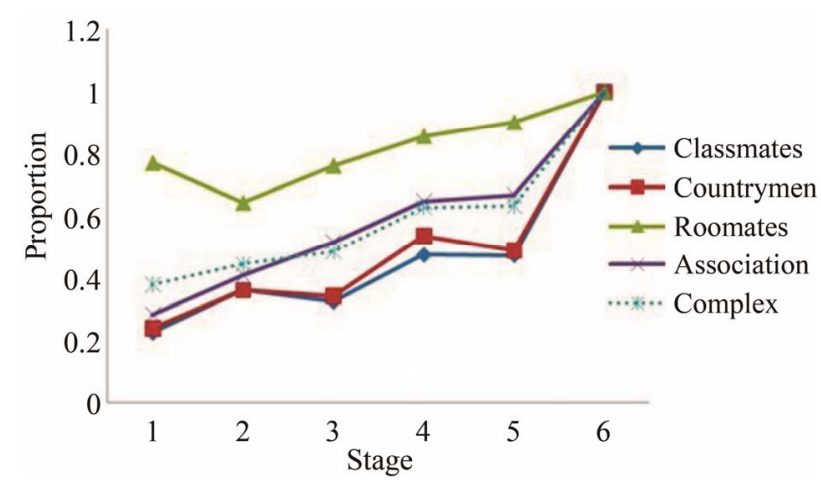

Figure 2. Average influence strength of love nodes in each stage.

then we call it the newly added love node in the $i+1$ th stage. In the corresponding subnet, the strength of node $B$ 's being affected by its neighbor nodes, in the ith stage, is given by (4). Here

$$
y_{B i}=N_{B i} / N_{B} .
$$

in which $y_{B i}$ means in a certain relationship subnet, node $B$ 's strength of being affected by its neighbor nodes in the $i$ th stage, $N_{B i}$ means the number of love nodes among the neighbors of node $B$ in the $i$ th stage, an $N_{B}$ represents the total number of all the neighbor nodes of node $B$ in the $i$ th stage.

At this point, we focus on all the newly added love nodes in the $i+1$ th stage, in a certain relationship subnet, the average strength of being affected by their neighbors in the ith stage is given by (2). Here

$$
\bar{y}_{i}=y_{i} / N_{n, i+1} \text {. }
$$

in which $\bar{y}_{i}$ means the average strength of being af- 
fected by their neighbors in the ith stage, $y_{i}$ means the summation of all the newly added love nodes' strengths of being affected in the ith stage, and $N_{n, i+1}$ represents the total number of the newly added love nodes in the $i+1$ th stage in the overall network. The study above, $y_{B i}$ and $\bar{y}_{i}$ are all carried out in a signal subnet.

At last, we study the overall situation of the whole network, and we focus on all the newly added love nodes in the $i+1$ th stage in the overall network. The average strength of being affected by their neighbors in the ith stage is given by (6). Here

$$
\bar{Y}_{i}=Y_{i} / N_{n, i+1} \cdot
$$

in which $\bar{Y}_{i}$ means the average strength of being affected by their neighbors in the ith stage, $Y_{i}$ means the summation of all the newly added love nodes' strengths of being affected in the ith stage in the whole network, and $N_{n, i+1}$ represents the total number of the newly added love nodes in the $i+1$ th stage in the overall network.

Formula (4) and Formula (5) are used to calculate according to the four subnets respectively, while Formula (6) is the comprehensive calculation of the whole campus social network.

According to Formula (5) and Formula (6), we got Figure 3 as below.

It can be seen from the figure above, both in each subnet and the whole network, the tendency of the average value of non-love nodes' strength of being affected is basically consistent, and substantially shows a rising trend as time goes, except for the two curve inflection point. As time goes by, more and more nodes become to love nodes, so the amount of love nodes out of one's neighbors is more and more, therefore the influence becomes greater.

So, in the whole university stage, counselors and teachers should attach great importance to guide the students to form a correct outlook on love. For instance, they can set up a specialized students love psychology course, and pay more attention to the emotional needs of the students and attach more importance to their state of love, work well for students.

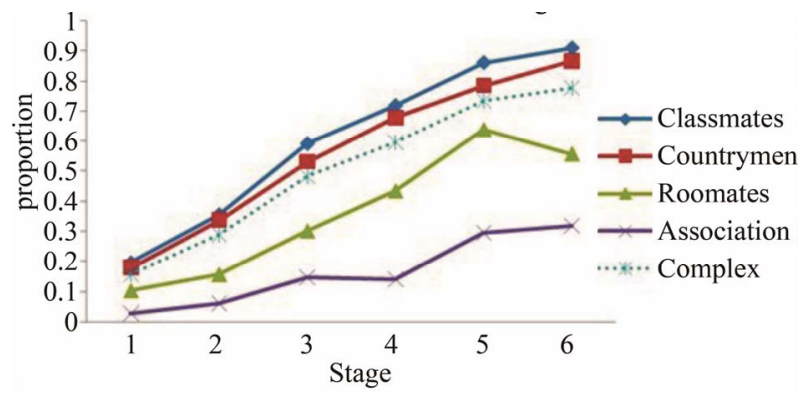

Figure 3. Non-love nodes' average strength of being affected in each stage.

\section{Statistical Analysis of the Spread of Amative Behavior in Kinds of Subnets}

In the process of the spread of amative behavior, nodes in the social network are affected by various aspects, for instance, classmates, countrymen, roommates and members of the same association. These relationships may have an impact on the node, may not produce any effect. Therefore, we first have a look to survey the subjective feeling of the investigated nodes about what kind of relationship it is that affected their love behavior most.

According to the questionnaire, we conducted a survey and obtained the influence factors in each. According to the stage results, we got Figure 4 as below.

As apparent from Figure 4, nearly half of the respondents believe that their love behavior is affected by "the whole atmosphere of the University”, which is a multiple rather than single social impact. It's the subjective feeling of the investigated nodes, while we did further analysis by objective data. By unifying the influence of the four subnets, we got the amount of the newly added love nodes influenced by the multiple factors in each stage, and the amount of the newly added love nodes influenced by one, two, three and four kinds of factors in each stage. Then we calculated the proportion of the latter accounted for of the former, and made a chart as Figure 5.

Figure $\mathbf{5}$ gives us the information conveyed is that nodes affected by a single relationship account for only a minority of the whole newly added love nodes in each stage, nodes affected by two and three kinds of factors account for the majority, and the proportion of the nodes affected by four kinds of influence factors accounted for of the all the newly added nodes is also gradually increased after the third stage. It shows that, in the process of spreading love behavior, the impact of a variety of social relationships is stronger than a single social relationship. The conclusion fits with the results of the survey. It also shows that the diversity of the social relationship between the love node and the non-love node plays the main role for the spread of the amative behavior.

\section{Conclusion}

In this paper, we carried out an empirical analysis of the

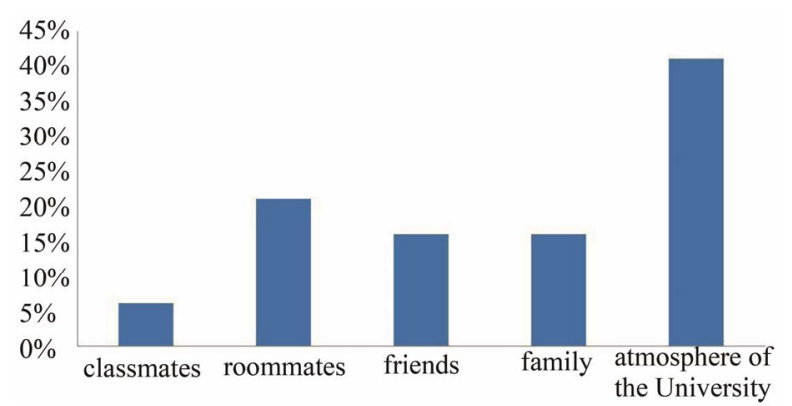

Figure 4. Nodes' subjective feelings of the influence factors. 


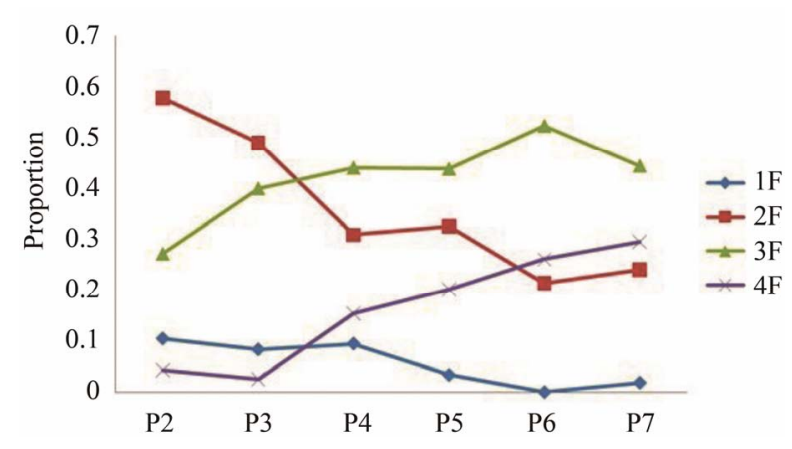

Figure 5. Proportion of different influence factors in each stage.

amative behavior of hundreds of seniors in a university campus. We found that the spread of amative behavior has different characteristics in different stage, which put forward suggestions on our students work. At the same time, we also found that the diversity of the social relationship plays the main role for the spread of the amative behavior.

\section{REFERENCES}

[1] P. Erdös and A. Rényi, “On Random Graphs,” Institute of the Hungarian Academy of Science, Vol. 5, No. 6, 1960, p. 17.
[2] D. J. Watts and S. H. Strogatz "Collective Dynamics of 'Small-World' Networks,” Nature, Vol. 393, No. 4, 1998, pp. 440-442.

[3] M. E. J. Newman and D. J. Watts, "Scaling and Percolation in the Small-World Network Model," Physical Review E, Vol. 60, No. 6, 1999, pp. 7332-7342. doi:10.1103/PhysRevE.60.7332

[4] M. Faloutsos, P. Faloutsos and C. Faloutsos, "On PowerLaw Relationships of the Internet Topology," Computer Communication Review, Vol. 29, No. 4, 1999, pp. 251262.

[5] Sen Petal, "Small-World Properties of the Indian Railway Network," Physical Review E, Vol. 67, No. 3, 2003, Article ID: 036106. doi:10.1103/PhysRevE.67.036106

[6] R. M. Anderson and R. M. May, "Infectious Diseases of Humans,” Oxford University Press, Oxford, 1992.

[7] P. S. Dodds, D. J. Watts and C. F. Sabel, "Information Exchange and the Robustness of Organizational Networks," Proceeding of the National Academy of Sciences of the United States of America, Vol. 100, No. 21, 2003, pp. 12516-12521.

[8] J. M. Kleinberg, "Navigation in a Small World," Nature, Vol. 406, No. 6798, 2000, p. 845.

[9] T. W. Valente, "Network Models of the Diffusion of Innovations," Computational and Mathematical Organization Theory, Vol. 2, No. 2, 1996, pp. 163-164. 